\title{
Long-term reproducibility of home vs. office blood pressure in children and adolescents: the Arsakeion school study
}

\author{
George S Stergiou, Efthimia G Nasothimiou, Periklis P Giovas and Vayia C Rarra
}

This study compared the long-term reproducibility of home blood pressure (BP) in comparison with office BP in children and adolescents. Forty-eight subjects (27 boys, mean age $11.3 \pm 3.1$ (s.d.) years) recruited from the Arsakeion school study because of elevated office and/or home BP were assessed with office (1 visit, mercury sphygmomanometer) and home BP measurements ( 3 days, electronic devices) in two assessments $17 \pm 4.9$ months apart (range 10-26 months). Home and office BP were compared on the basis of the following criteria: (a) s.d. of mean BP; (b) s.d. of differences; (c) variation coefficient (CV); (d) concordance correlation coefficient (CCC); (e) test-retest correlations; (f) correlation with ambulatory BP. (a) The s.d. of mean home BP was lower than that of office BP in both the initial (home BP 9.1/7.1 mm Hg, systolic/diastolic; office BP 13.1/ $8.0 \mathrm{~mm} \mathrm{Hg}$ ) and the second assessment $(9.2 / 6.0$ and 14.9/11.5 respectively). (b) The s.d. of differences was lower for home BP $(8.3 / 6.5 \mathrm{~mm} \mathrm{Hg}$, systolic/diastolic) than for office BP $(13.9 / 10.7 \mathrm{~mm} \mathrm{Hg})$. (c) The CV of home BP $(5.3 / 6.6$, systolic/diastolic) was lower than that of office BP (8.2/10.9). (d) The CCC of home BP (0.54/0.50, systolic/diastolic) was higher than that of office BP (0.51/0.41). (e) Test-retest correlations were closer for home BP ( $r=0.58 / 0.52$, systolic/diastolic) than for office BP (0.51/0.44). (f) Awake ambulatory BP was more closely associated with home ( $r=0.77 / 0.40$, systolic/diastolic) than with office BP $(0.65 / 0.24)$. These data suggest that in children and adolescents the long-term reproducibility of home BP is superior to that of office measurements.

Hypertension Research (2009) 32, 311-315; doi:10.1038/hr.2009.9; published online 13 February 2009

Keywords: children; home blood pressure; repeatability; reproducibility; self-measurement

\section{INTRODUCTION}

Current guidelines in Europe and the United States recommend the wide use of home blood pressure (BP) monitoring in the adults for the detection of the white coat and the masked hypertension phenomena and the long-term follow-up of treated hypertension. ${ }^{1-3}$ In children and adolescents, the white coat and masked hypertension phenomena are not uncommon and therefore out-of-office BP monitoring is often needed. ${ }^{4-6}$ Studies have shown the usefulness of ambulatory BP monitoring in the pediatric population, ${ }^{7}$ and a statement by the American Heart Association for the application of this method in children has been recently published. ${ }^{8}$ However, there is little evidence on the usefulness of home BP monitoring in children.

The reproducibility of any measurement method is an essential feature deserving thorough assessment before its wide application in clinical practice. Multiple studies have shown that the conventional measurements of BP by physicians in the office have poor reproducibility, and random BP changes from visit to visit might often exceed the BP changes achieved by an effective antihypertensive drug. ${ }^{1,9-11}$ On the other hand, ambulatory BP measurements are more reproducible than office measurements in the adults, ${ }^{1,9-11}$ and also in children. ${ }^{8,12-15}$ Regarding the home BP measurements, studies in adults have shown that these are more reproducible than office measurements and as reproducible as ambulatory BP..$^{1-3,10,16,17}$ In children and adolescents, one small short-term study again showed the reproducibility of home BP to be superior to office measurements and as good as of ambulatory BP. ${ }^{14}$

The objective of this study is to assess the long-term reproducibility of home BP in children and adolescents in comparison with the conventional office measurements.

\section{METHODS}

Subjects and study design

This prospective nested study enrolled children and adolescents from the Arsakeion study, a school-based cross-sectional study that evaluated home and office BP measurements in 778 healthy children and adolescents aiming to determine the normal range of home $\mathrm{BP}$ in the pediatric population. ${ }^{18}$ Exclusion criteria were current or previous treatment with antihypertensive drugs, diabetes mellitus, and renal, cardiac, or other systemic disease or acute

Third University Department of Medicine, Hypertension Center, Sotiria Hospital, University of Athens, Athens, Greece This paper was presented at the 22nd International and 18th European Society of Hypertension Meeting, Berlin 2008.

Correspondence: Dr GS Stergiou, Third University Department of Medicine, Hypertension Center, Sotiria Hospital, University of Athens, 152 Mesogion Avenue, Athens 11527 , Greece. 
illness. Participants who were invited to be re-evaluated in a 'second assessment' should have office BP > 90th percentile for height, age, and gender ${ }^{7}$ and/or home BP $\geqslant 95$ th percentile for height and age ${ }^{18}$ in the Arsakeion study ('first assessment') and accept the invitation to be re-examined. A medical history was taken with the assistance of the participants' parents, and anthropometric characteristics (weight, height, arm circumference) were recorded in both assessments. Written informed consent was obtained from all participants and/or their parents, and the protocol was approved by the hospital scientific committee.

\section{Blood pressure measurements}

Office BP measurements were taken in a quiet room in the school building by three physicians who fulfilled the British Hypertension Society Protocol criteria for observer agreement in BP measurement ${ }^{19}$ and were experienced in BP evaluation in children. Duplicate measurements were taken in a single visit in the first and the second assessment after 5 min sitting rest and with at least $1 \mathrm{~min}$ between recordings using a standard mercury sphygmomanometer (cuffs with inflatable bladder $9 \times 18 \mathrm{~cm}$ (small), $12 \times 23 \mathrm{~cm}$ (medium), or $15 \times 35 \mathrm{~cm}$ (large) to cover $80-100 \%$ of the individual's arm circumference, Korotkoff phase $\mathrm{V}$ for diastolic BP, or phase IV when sounds could be heard to $0 \mathrm{~mm} \mathrm{Hg})^{7}$

Home BP was monitored for three routine school days within a week ${ }^{20}$ using validated automated oscillometric devices Omron 705IT (Omron Healthcare Europe BV, Hoofddorp, The Netherlands; inflatable bladder size $9 \times 16,13 \times 23$, or $15 \times 30 \mathrm{~cm}$ where appropriate), ${ }^{21}$ in both the first and the second assessment. Participants or their parents for younger children were trained in the conditions of home BP measurement and the use of the electronic devices. They were instructed to take duplicate morning (06:00-10:00 hours) and evening (18:00-22:00 hours) home BP measurements after 5 min sitting rest and with 1 min interval between measurements. A form was supplied to the participants to report all their home BP values, which were also downloaded from the devices through a computer link.

In the second assessment, participants were also evaluated with ambulatory BP monitoring using validated oscillometric devices SpaceLabs 90207 or 90217 (SpaceLabs Inc., Redmond, WA, USA; bladder size $9 \times 16$ or $12 \times 23 \mathrm{~cm}$ where appropriate) were used..$^{22,23}$ The monitors were programmed to measure BP at 20 -min intervals for $24 \mathrm{~h}$ and were applied on a routine school day. Subjects were instructed to follow their usual daily activities but to avoid extremely strenuous physical activities and to remain still with the forearm extended during each BP reading. A brief diary specifying the time when they went to bed and arose was obtained by all participants. All office, home, and ambulatory BP measurements were taken in the same (non-dominant) arm of each individual. Before each home or ambulatory BP monitoring session, the accuracy of the device was tested against a standard mercury sphygmomanometer in each individual (three succeeding readings; $\mathrm{Y}$ connector) to ensure that there was no consistent difference of $>10 \mathrm{~mm} \mathrm{Hg}$ in measured BP.

\section{Diagnostic thresholds and definitions}

Office hypertension and pre-hypertension were defined as systolic and/or diastolic BP $\geqslant 95$ th percentile and $>90$ percentile respectively, on the basis of the 2004 NHBPEP Report office BP normalcy tables. ${ }^{7}$ Ambulatory hypertension was diagnosed on the basis of normative values of the German Working Group on Pediatric Hypertension ${ }^{23}$ and home hypertension (systolic and/or diastolic BP $\geqslant 95$ th percentile) using the normative date from the Arsakeion School study. ${ }^{18}$ Sustained hypertension was defined as elevated office and awake ambulatory or home BP, white-coat hypertension as elevated office BP with normal awake ambulatory or home $\mathrm{BP}$, and masked hypertension the reverse. ${ }^{1,4,5,7,8}$

\section{Analysis}

Participants who provided fewer than six valid home BP readings were excluded. For each assessment all valid home BP readings were averaged to give a single number per individual. Ambulatory BP recordings with fewer than 20 successful awake readings were excluded. Measurements flagged by the software of the monitors as being technically erroneous were excluded. Ambulatory or home measurements with systolic BP $<50$ or $>260 \mathrm{~mm} \mathrm{Hg}$ and those with diastolic $<30$ or $>150 \mathrm{~mm} \mathrm{Hg}$ were also excluded, as well as early readings taken less than $20 \mathrm{~min}$ after the monitor had been attached to subjects, because these had been taken in the clinic. The average of awake ambulatory BP was used in the analysis, which was calculated using the individuals' sleeping times. The average of duplicate office BP readings of each assessment was used for analysis.

The following criteria were used to assess the stability (a), reproducibility (b-e), and reliability (f) of office and home BP measurements:

(a) s.d. of average BP;

(b) s.d. of the differences between repeated BP measurements with BlandAltman scatterplots;

(c) coefficient of variation for repeated measurements (mean of the s.d. values of the differences between repeated measurements divided by the mean $\mathrm{BP}$ of the repeated measurements of the paired sample);

(d) concordance correlation coefficient (CCC) $(r \times \mathrm{Cb}$, where $r$ is the Pearson correlation coefficient measuring how far each observation deviates from the best-fit line and $\mathrm{Cb}$ a bias correction factor measuring how far the best-fit line deviates from the $45^{\circ}$ line through the origin); ${ }^{24}$

(e) test-retest correlations evaluating the relationship between repeated BP measurements;

(f) correlation with awake ambulatory BP.

Student's paired $t$-tests were used for the comparison of repeated BP measurements, and Pearson correlations coefficients $(r)$ for test-retest correlations and the assessment of the association of office and home BP with ambulatory BP. F-test was used for the statistical comparison of s.d. values. The statistical analysis was performed using the Minitab statistical software (release 13.31; Minitab Inc., State College, PA, USA). A probability value $P<0.05$ was considered statistically significant.

\section{RESULTS}

\section{Subjects}

A total of 96 children fulfilled the study criteria (elevated office and/or home BP) in the initial assessment of the Arsakeion study and all of them were invited to participate. Fifty-one subjects and their parents agreed to participate and were enrolled in the study. The main reasons why 45 of the 96 children refused to be re-evaluated were parents' unconcern about BP $(n=16)$, lack of contact details because of school graduation $(n=9)$, parents' preference to consult their family doctor $(n=5)$, parents' or children's lack of time $(n=5)$, children refused $(n=2)$, and other reasons $(n=6)$. No statistically significant differences were found between children enrolled in the study and those who refused to participate regarding their age, gender, height, weight, body mass index, family history of hypertension, number of home BP readings, and office and home BP levels in the first assessment. Three subjects were excluded because of inadequate home BP monitoring and the rest 48 were included in the analysis ( 27 boys, mean age $11.3 \pm 3.1$ (s.d.) years, range 6.2-17.5 years). The average arm circumference was $23.0 \pm 4.0 \mathrm{~cm}$ (range $16-32 \mathrm{~cm}$ ). The small size cuff was used in 22 participants $(46 \%)$ and the medium in $26(54 \%)$. The mean time interval between the first and the second assessment was $17 \pm 4.9$ months (range 10-26 months). As expected, there were significant increases in age, height, weight and body mass index from the first to the second assessment (Table 1).

\section{Blood pressure measurements}

In the first assessment, office BP was higher than the 95th percentile ${ }^{7}$ in 17 subjects (35\%) and within 90-95th percentile in $8(17 \%)$, and in the second assessment, $18(37.5 \%)$ and $5(10 \%)$, respectively. About $90 \%$ of the participants provided more than 10 of the 12 requested home BP readings in both assessments (average number of obtained readings $11.4 \pm 1.7$ (s.d.) in the first and $11.6 \pm 1.4$ the second 
Table 1 Participants' anthropometric characteristics and blood pressure measurements during the first and the second assessment (mean \pm s.d.)

\begin{tabular}{lccc}
\hline & Assessment A & Assessment B & P-value (A vs. B) \\
\hline Age (years) & $11.3 \pm 3.1$ & $12.7 \pm 2.8$ & $<0.001$ \\
Height (cm) & $154.0 \pm 19.8$ & $158.8 \pm 17.0$ & $<0.001$ \\
Weight (kg) & $51.4 \pm 21.0$ & $57.3 \pm 21.2$ & $<0.001$ \\
Body mass index $\left(\mathrm{kg} \mathrm{m}^{-2}\right)$ & $20.7 \pm 4.5$ & $22.0 \pm 5.0$ & $<0.001$ \\
OBP systolic $\left(\mathrm{mm} \mathrm{Hg}^{-}\right.$ & $118.0 \pm 13.1^{*}$ & $120.0 \pm 14.9$ & $\mathrm{NS}$ \\
OBP diastolic $(\mathrm{mm} \mathrm{Hg})$ & $70.5 \pm 8.0$ & $68.7 \pm 11.5$ & $\mathrm{NS}$ \\
HBP systolic $(\mathrm{mm} \mathrm{Hg})$ & $121.9 \pm 9.1$ & $118.2 \pm 9.2$ & $<0.01$ \\
HBP diastolic $(\mathrm{mm} \mathrm{Hg})$ & $70.0 \pm 7.1$ & $68.8 \pm 6.0$ & $\mathrm{NS}$ \\
ABP systolic $(\mathrm{mm} \mathrm{Hg})$ & - & $120.6 \pm 11.5^{*}$ & - \\
ABP diastolic $(\mathrm{mm} \mathrm{Hg})$ & - & $70.6 \pm 5.5$ & -
\end{tabular}

Abbreviations: ABP, awake ambulatory blood pressure; HBP, home blood pressure; NS, not significant; OBP, office blood pressure.

${ }^{*} P<0.05$, for comparison vs. HBP of the same column

Table 2 Criteria of stability (a), reproducibility (b-e), and reliability (f) of office and home blood pressure measurements in children and adolescents

\begin{tabular}{lcc}
\hline Criteria & $\begin{array}{c}\text { Home } \\
\text { Office }\end{array}$ \\
\hline (a) s.d. (assessment A) & BP (systolic/diastolic) & BP (systolic/diastolic) \\
s.d. (assessment B) & $9.1 / 7.1$ & $13.1 / 8.0$ \\
(b) s.d. of differences & $9.2 / 6.0$ & $14.9 / 11.5$ \\
(c) Variation coefficient (\%) & $8.3 / 6.5$ & $13.9 / 10.7$ \\
(d) CCC & $5.3 / 6.6$ & $8.2 / 10.9$ \\
(e) Test-retest, $r$ & $0.54 / 0.50$ & $0.51 / 0.41$ \\
(f) $r$ with ambulatory BP & $0.58 / 0.52$ & $0.51 / 0.44$ \\
\hline
\end{tabular}

Abbreviations: BP, blood pressure; CCC, concordance correlation coefficient; $r$, correlation coefficient; s.d., standard deviation of mean BP; assessment A/B, first/second assessment.

assessment). In the first assessment, home BP was $>95$ th percentile ${ }^{18}$ in 22 participants $(46 \%)$ (16 (33\%) with low office BP, suggesting masked hypertension) and in the second assessment in 11 participants $(24 \%)$ (6 (11\%) with low office BP). Office BP did not change significantly between the two assessments (mean difference systolic $-1.9 \pm 13.9 \mathrm{~mm} \mathrm{Hg}, 95 \%$ confidence intervals (CI) $-5.9,2.2$ and diastolic $1.8 \pm 10.7 \mathrm{~mm} \mathrm{Hg}, 95 \% \mathrm{CI}-1.4,4.9$ ) (Table 1). Diastolic home BP was also unchanged between the two assessments (difference $1.2 \pm 6.5,95 \%$ CI $-0.7,3.0$ ) (Table 1), yet systolic home BP was lower in the second assessment by $3.8 \pm 8.3,95 \%$ CI 1.4, 6.2, $P<0.01$ ). Systolic home BP was lower by $2.5 \pm 7.8 \mathrm{~mm} \mathrm{Hg}$ (95\% CI 0.18, 4.7, $P<0.05)$ than awake ambulatory BP of the second assessment, with no such difference in diastolic BP (Table 1).

Criteria of stability (a) reproducibility (b-e) and reliability (f) of blood pressure measurements (Table 2):

(a) The s.d. of mean home BP was consistently lower than that of office BP, in both the initial $(P<0.05 / 0.42$, systolic/diastolic) and the second $(P<0.01 / 0.001)$ assessment.

(b) The s.d. of differences between repeated home BP was lower than that of office measurements $(P<0.01$ for both systolic and diastolic). The reproducibility of home to office BP measurements is also illustrated with the Bland-Altman scatterplots (Figures 1 and 2). The s.d. of differences of the first two home BP readings was $10.9 / 12.7 \mathrm{~mm} \mathrm{Hg}$ (systolic/diastolic), of the morning home BP readings $(n=6)$, it was $10.0 / 8.9 \mathrm{~mm} \mathrm{Hg}$, of the evening
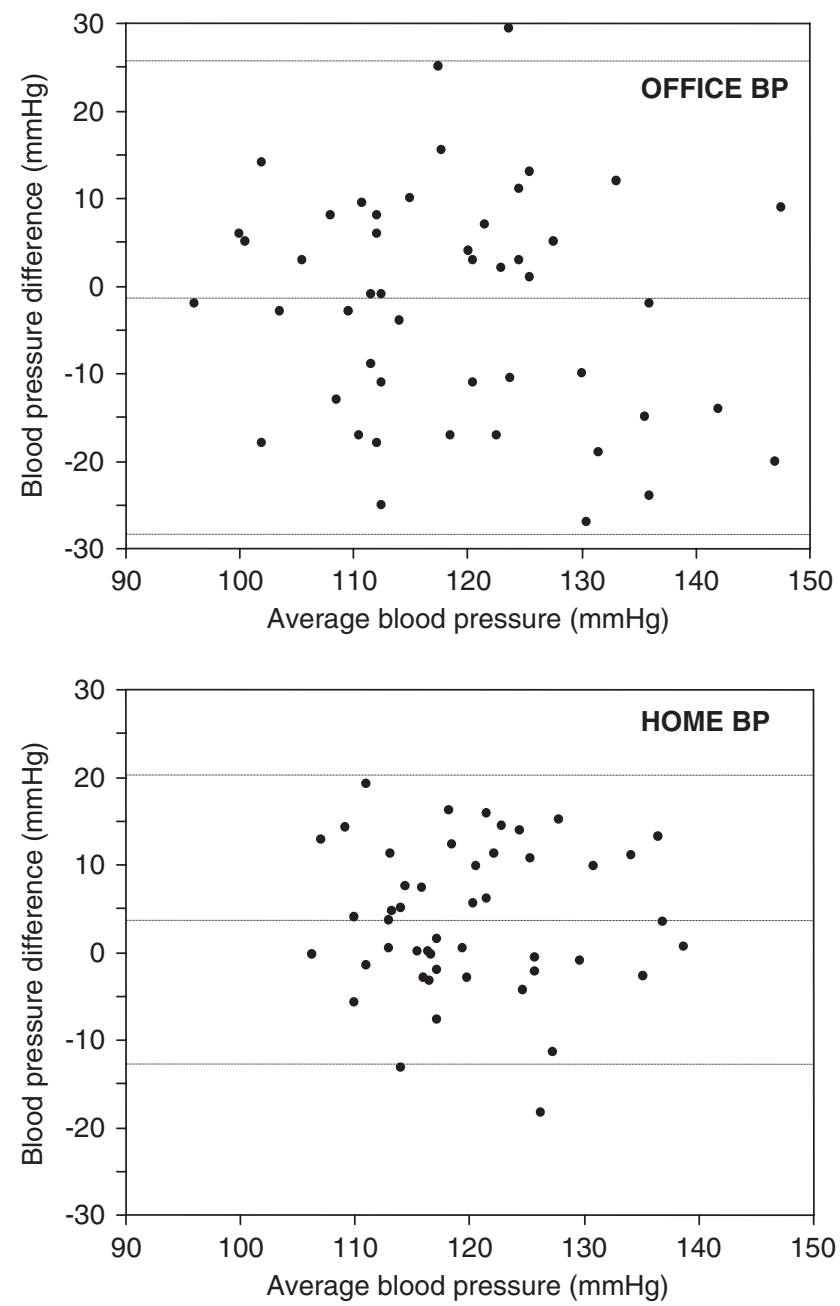

Figure 1 Discrepancies for the Bland-Altman technique between repeated office and home systolic blood pressure (BP) measurements. Horizontal lines indicate mean differences between repeated measurements and limits of agreement ( \pm 2 s.d.) within which $95 \%$ of the differences are expected to lie.

readings $(n=6)$, it was $9.9 / 6.8 \mathrm{~mm} \mathrm{Hg}$, of the total of first measurements per occasion $(n=6)$, it was $8.9 / 7.0 \mathrm{~mm} \mathrm{Hg}$, and of the first day's home readings $(n=4)$, it was $8.9 / 7.8 \mathrm{~mm} \mathrm{Hg}$.

(c) Coefficients of variation were again lower for home than for office BP, systolic and diastolic.

(d) Concordance correlation coefficients were also consistently higher for home than for office BP, systolic and diastolic.

(e) Test-retest correlation coefficients were again consistently higher for home than for office BP, systolic and diastolic.

(f) Correlations of awake ambulatory BP with home BP were stronger than with office measurements, systolic and diastolic.

\section{DISCUSSION}

This study in children and adolescents compared the reproducibility of home BP with office measurements on repeated assessments 17 months apart. Across all the criteria applied, home BP appeared to be consistently superior to the office measurements for both systolic and diastolic BP.

To our knowledge this is the first study in children and adolescents comparing the long-term measurement error in home BP monitoring against the conventional office measurements. In both assessments, 

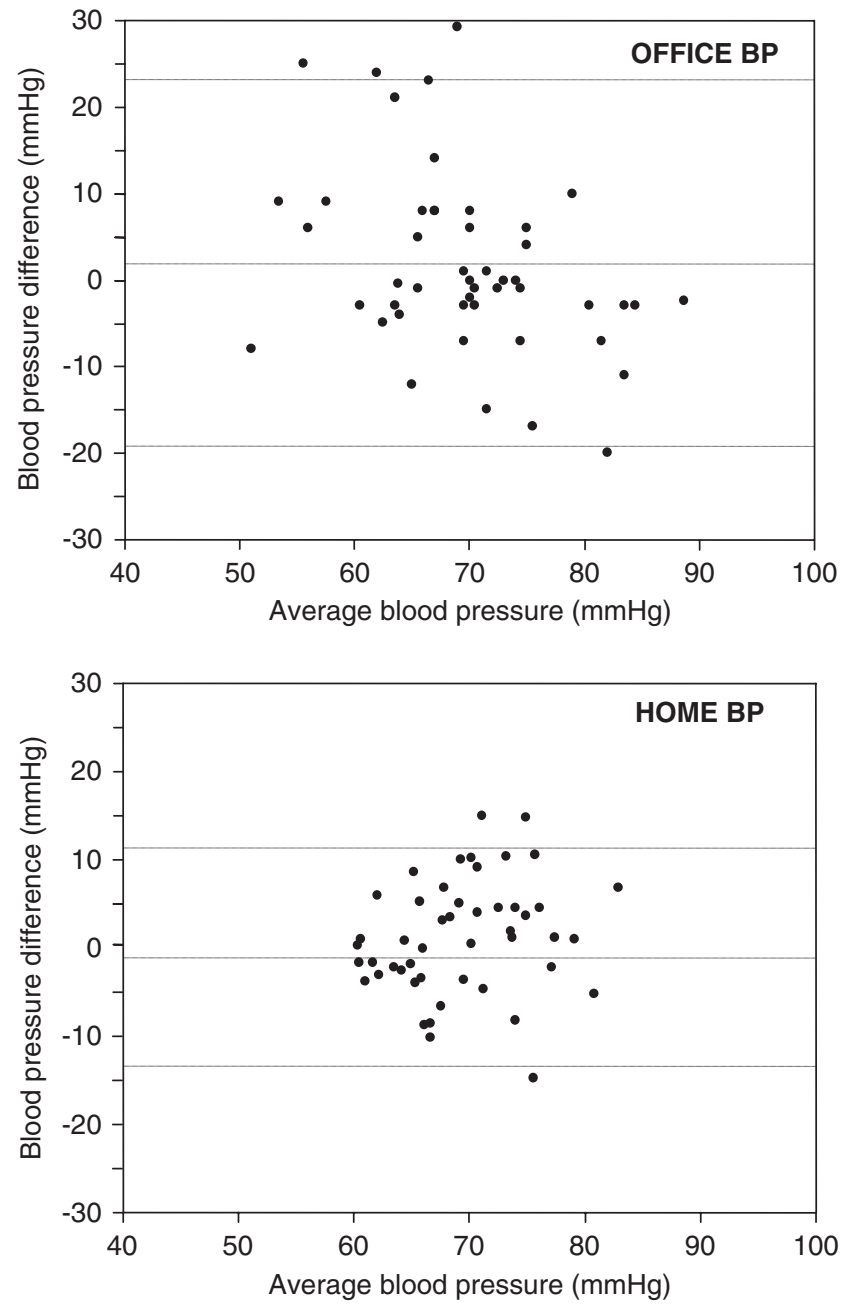

Figure 2 Discrepancies for the Bland-Altman technique between repeated office and home diastolic blood pressure (BP) measurements. Horizontal lines indicate mean differences between repeated measurements and limits of agreement ( \pm 2 s.d.) within which $95 \%$ of the differences are expected to lie.

office BP was measured by well-trained observers in the same setting and conditions, and home BP was measured by using an automated device that has been previously validated in children and adolescents. $^{21}$ The potential of reporting bias by children regarding their home readings was prevented by using devices with automated memory and PC link capacity, and a monitoring schedule that has been reported previously to provide a reliable assessment of BP at home in children and adolescents has been used. ${ }^{20}$

Several different criteria have been applied to test the reliability and the level of measurement error between repeated assessments, because none of them alone can fully assess the reproducibility characteristics. ${ }^{25}$ Measurement error defined as the variation between measurements of the same quantity on the same individual can be assessed by the s.d. of the mean and the s.d. of differences between repeated measurements. ${ }^{25}$ Indeed, office BP had a higher variability (s.d. of mean) than home BP in both assessments, and also lower reproducibility (higher s.d. of differences between assessments). Another reliable reproducibility index is the variation coefficient that depends on both the s.d. of the differences and the mean value of the pairwise repeated BP measurements, which was again superior for home BP. The measurement error can also be assessed by the CCC, which is a measure of precision and accuracy and evaluates the degree to which pairs of observations fall on the $45^{\circ}$ line through the origin. ${ }^{24}$ The testretest correlation coefficient is a poor index of reproducibility, but only determines the strength of linear association between two assessments and complements the other criteria. Furthermore, the association with ambulatory BP, which is regarded as the optimal method for the assessment of the BP load on the cardiovascular system, ${ }^{1,8}$ was again stronger for home measurements. What is important in this study is that all the above criteria consistently show to the same direction that is in favour of home BP measurements.

In the adults, the short-term reproducibility of home BP appears to be as good as of ambulatory BP and better than that of office measurements. ${ }^{10,16,17}$ In children and adolescents only one small study ( $n=16)$ has assessed the short-term reproducibility ( 8 weeks) of home compared with office BP. ${ }^{14}$ In this study conducted in a research setting, both office and home measurements were more reproducible than in the present study, yet again home BP was superior to office measurements (home BP: s.d. of differences 7.0/ 4.3, systolic/diastolic and test-retest $r=0.74 / 0.82$; office BP: $10.4 / 6.3$ and $0.63 / 0.80$, respectively). ${ }^{14}$

Regarding the long-term reproducibility of home BP limited data exist in the adults. Sakuma et al. ${ }^{17}$ compared the reproducibility of home with office BP over 1-year period and showed home BP to be superior (home BP: s.d. of differences 7.7/5.5 systolic/diastolic; office BP: $13.8 / 10.2$, respectively). These data are nearly identical with the present findings in children and adolescents and suggest similar reproducibility of home BP in adults and in children. This advantage of home over office BP is probably due to the fact that these measurements are taken in the usual environment of each individual and to the larger number of measurements averaged. Yet, the larger number of measurements might be more important, given that the better reproducibility of home BP was not present when equal number of office and home measurement was used $(n=2)$. Furthermore, when the reproducibility of the average home BP of all 12 readings was compared with that of different home BP values, such as morning home BP, evening, single readings per occasion, and first day's readings, neither of these aspects of home BP was superior to the average based on a larger number of readings $(n=12)$.

Lurbe et al., ${ }^{12}$ investigated the long-term reproducibility of ambulatory BP in 30 healthy children assessed 7 months apart and reported s.d. of difference, 4.9-5.8/3.3-4.0 for systolic/diastolic. In another study by the same investigators in 31 children assessed twice 4 months apart, the s.d. of differences was 5.9/5.5 for daytime ambulatory BP. ${ }^{13}$ The long-term reproducibility of daytime ambulatory BP assessed over a 2-year period in 18 stable children after renal transplantation was similar to that of home BP reported in this study and superior to that of office measurement (s.d. of differences 7.1/5.5, systolic/diastolic for ambulatory BP vs. 13.7/9.5 for office BP). ${ }^{15}$ The only study in children and adolescents that provided a direct comparison of the reproducibility (short-term) of home vs. ambulatory BP reported a s.d. of differences for home BP 7.0/4.3 and for ambulatory BP 5.9/5.0. ${ }^{14}$ Taken together, these data suggest that in the pediatric population the short-term and long-term reproducibility of home BP is similar to that of ambulatory BP and higher than that of the conventional office measurements.

In children and adolescents, the yearly rise in BP is modified by age, gender, and height. The expected rise in office BP between assessments $\mathrm{A}$ and $\mathrm{B}$ in the study population could be estimated on the basis of the 50th percentile of BP of each individual participant taken from normalcy tables, which gives a real sense of the midpoint of the 
normal range of BP. ${ }^{7}$ The expected mean office BP was $105.9 \pm 6.6 /$ $61.9 \pm 3.0 \mathrm{~mm} \mathrm{Hg}$ in assessment A and $108 \pm 6.4 / 63.1 \pm 2.7 \mathrm{~mm} \mathrm{Hg}$ in assessment B. Thus, the expected BP change during the study follow-up would be $2.4 \pm 2.8 / 1.3 \pm 1.3 \mathrm{~mm} \mathrm{Hg}$ for systolic/diastolic $(P<0.001)$. In this study, the $95 \% \mathrm{CI}$ of the difference in office BP between the two assessments ( $-5.9,2.2 /-1.4,4.9$ for systolic/diastolic) include the expected change in BP as calculated above. Besides, any factors influencing the BP change with increasing age in this study are expected to affect equally office and home BP. Thus, the comparison of the reproducibility of the two measurement methods (office $v s$. home BP) is not affected by such confounders.

Despite the increase in the participants' body size from the first to the second assessment in this study, systolic home BP was reduced in the second assessment with no such change in diastolic home or in office BP (Table 1). We do not have a plausible explanation for this finding given that such a decrease in home BP in repeated measurements has not been previously observed in adults ${ }^{10,16,17}$ or in children. ${ }^{14}$ According to the home BP normalcy data derived from the same study in the total population of 778 children and adolescents, the expected change in systolic home BP would be an increase by $2-3 \mathrm{~mm} \mathrm{Hg} .{ }^{18}$ A regression to the mean effect or chance finding due to small study sample might be a possible explanation. It should be mentioned that the unbiased recording of home measurements in this study and the identical s.d. of the mean systolic home BP in the two assessments imply that reliable home measurements have been obtained. Regarding the reversed relationship between home and office $\mathrm{BP}$ in the assessment A vs. B, an actual change in this relationship with increasing age in children might have really occurred. In a previous analysis of the total population of the Arsakeion study $(n=778),{ }^{26}$ age was significantly correlated with the office BP-home BP difference $(r=0.13 / 0.24$ for systolic/diastolic, $P<0.001)$. In the younger children (6-12 years) home BP was higher than office BP, whereas in the older children and in adolescents this BP difference was eliminated. ${ }^{26}$ This study, with mean age 11.3 years in assessment $A$ and 12.7 years in assessment $B$, is in line with the change in the officehome BP relationship after the age of 12 years. ${ }^{26}$ Further studies are needed to confirm this trend. ${ }^{26}$

In conclusion, in children and adolescents the long-term reproducibility of home BP obtained using validated automated devices appears to be superior to that of carefully taken office measurements. Further research is needed to confirm these findings and establish the role of home BP monitoring in the diagnosis and management of pediatric hypertension.

\section{ACKNOWLEDGEMENTS}

This study was supported by the University of Athens Grant (Kapodistrias Program 2005 and 2006, Grant no. 70/4/8107). Home blood pressure monitors were provided by G Leoussis IS, Athens, Greece.

\section{CONFLICT OF INTEREST}

None.

1 O'Brien E, Asmar R, Beilin L, Imai Y, Mallion JM, Mancia G, Mengden T, Myers M, Padfield P, Palatini P, Parati G, Pickering T, Redon J, Staessen J, Stergiou G, Verdecchia $P$, on behalf of the European Society of Hypertension Working Group on Blood Pressure Monitoring. European Society of Hypertension recommendations for conventional, ambulatory and home blood pressure measurement. J Hypertens 2003; 21: 821-848.

2 Pickering TG, Miller NH, Ogedegbe G, Krakoff LR, Artinian NT, Goff D, American Heart Association. American Society Of Hypertension; Preventive Cardiovascular Nurses Association: Call to action on use and reimbursement for home blood pressure monitoring: executive summary: a joint scientific statement from the American Heart
Association, American Society Of Hypertension, and Preventive Cardiovascular Nurses Association. Hypertension 2008; 52: 1-9.

3 Parati G, Stergiou GS, Asmar R, Bilo G, de Leeuw P, Imai Y, Kario K, Lurbe E, Manolis A, Mengden T, O'Brien E, Ohkubo T, Padfield P, Palatini P, Pickering T, Redon J, Revera M, Ruilope LM, Shennan A, Staessen JA, Tisler A, Waeber B, Zanchetti A, Mancia G. ESH Working Group on Blood Pressure Monitoring. European Society of Hypertension guidelines for blood pressure monitoring at home: a summary report of the Second International Consensus Conference on Home Blood Pressure Monitoring. J Hypertens 2008; 26: 1505-1526.

4 Stergiou GS, Yiannes NJ, Rarra VC, Alamara CV. White-coat hypertension and masked hypertension in children. Blood Press Monit 2005; 10: 297-300.

5 Lurbe E, Torro I, Alvarez V, Nawrot T, Paya R, Redon J, Staessen JA. Prevalence, persistence, and clinical significance of masked hypertension in youth. Hypertension 2005; 45: 493-498.

6 Stergiou GS, Nasothimiou E, Giovas P, Kapoyiannis A, Vazeou A. Diagnosis of hypertension in children and adolescents based on home versus ambulatory blood pressure monitoring. J Hypertens 2008; 26: 1556-1562.

7 National High Blood Pressure Education Program Working Group on High Blood Pressure in Children and Adolescents. The fourth report on the diagnosis, evaluation, and treatment of high blood pressure in children and adolescents. Pediatrics 2004; 114: 555-576.

8 Urbina E, Alpert B, Flynn J, Hayman L, Harshfield GA, Jacobson M, Mahoney L, McCrindle B, Mietus-Snyder M, Steinberger J, Daniels S. American Heart Association Atherosclerosis, Hypertension, and Obesity in Youth Committee American Heart Association Atherosclerosis, Hypertension, and Obesity in Youth Committee: ambulatory blood pressure monitoring in children and adolescents: recommendations for standard assessment: a scientific statement from the American Heart Association Atherosclerosis, Hypertension, and Obesity in Youth Committee of the council on cardiovascular disease in the young and the council for high blood pressure research. Hypertension 2008; 52: 433-451.

9 Coats A, Radaelli A, Clark S, Conway J, Sleight P. The influence of ambulatory blood pressure monitoring on the design and interpretation of trials in hypertension. J Hypertens 1992; 10: 385-391.

10 Stergiou GS, Baibas NM, Gantzarou AP, Skeva II, Kalkana CB, Roussias LG, Mountokalakis TD. Reproducibility of home, ambulatory and clinic blood pressure: implications on the design of trials for the assessment of antihypertensive drug efficacy. Am J Hypertens 2002; 15: 101-104.

11 Brueren MM, van Limpt P, Schouten HJA, Leeuw PW, van Ree JW. Is a series of blood pressure measurements by the general practitioner or the patient a reliable alternative to ambulatory blood pressure measurements? A study in general practice with reference to short-term and long-term between-visit variability. Am J Hypertens 1997; 10: 879-885.

12 Lurbe E, Aguilar F, Gomez A, Tacons J, Alvarez V, Redon J. Reproducibility of ambulatory blood pressure monitoring in children. J Hypertens 1993; 11: S288-S289.

13 Lurbe E, Thijs L, Redón J, Alvarez V, Tacons J, Staessen J. Diurnal blood pressure curve in children and adolescents. J Hypertens 1996; 14: 41-46.

14 Stergiou GS, Alamara CV, Salgami EV, Vaindirlis IN, Dacou-Voutetakis C, Mountokalakis TD. Reproducibility of home and ambulatory blood pressure in children and adolescents. Blood Press Monit 2005; 10: 143-147.

$15 \mathrm{Krmar}$ RT, Berg UB. Long-term reproducibility of routine ambulatory blood pressure monitoring in stable pediatric renal transplant recipients. Am J Hypertens 2005; 18: 1408-1414

16 Mengden T, Battig B, Vetter W. Self-measurement of blood pressure improves the accuracy and reduces the number of subjects in clinical trials. J Hypertens 1991; 9: S336-S337.

17 Sakuma M, Imai Y, Nagai K, Watanabe N, Sakuma H, Minami N, Satoh H, Abe K. Reproducibility of home blood pressure measurements over a 1-year period. Am J Hypertens 1997; 10: 798-803.

18 Stergiou GS, Yiannes NG, Rarra VC, Panagiotakos DB. Home blood pressure normalcy in children and adolescents: the Arsakeion School study.J Hypertens 2007; 25: 1375-1379.

19 O'Brien E, Petrie J, Littler W, de Swiet M, Padfield PL, Altman DG, Bland M, Coats A, Atkins N. An outline of the revised British Hypertension Society protocol for the evaluation of blood pressure measuring devices. J Hypertens 1993; 11: 677-679.

20 Stergiou G, Christodoulakis G, Giovas P, Lourida P, Alamara C. Home blood pressure monitoring in children: how many measurements are needed? Am J Hypertens 2008; 21: 633-638.

21 Stergiou GS, Yiannes NG, Rarra VC. The Arsakion school study: validation of the Omron 705 IT oscillometric device for home blood pressure measurement in children and adolescents. Blood Press Monit 2006; 11: 229-234.

22 Belsha CW, Wells TG, Rice HB, Neaville WA, Berry PL. Accuracy of the Spacelabs 90207 ambulatory blood pressure monitor in children and adolescents. Blood Press Monit 1996; 1: 127-133.

23 Soergel M, Kirschstein M, Busch C, Danne T, Gellermann J, Holl R, Krull F, Reichert H, Reusz GS, Rascher W. Oscillometric twenty-four-hour ambulatory blood pressure values in healthy children and adolescents: a multicenter trial including 1141 subjects. J Pediatr 1997; 130: 178-184.

24 Bland JM, Altman DG. Measurement error. BMJ 1996; 313: 744-753.

25 Lin L. A concordance correlation coefficient to evaluate reproducibility. Biometrics 1989; 45: 255-268.

26 Stergiou GS, Rarra VC, Yiannes NG. Changing relationship between home and office blood pressure with increasing age in children: the Arsakeion School study. Am J Hypertens 2008; 21: 41-46. 